\title{
A CHARACTERIZATION OF ORBITS
}

JAMES W. ENGLAND

In this paper we give a necessary and sufficient condition in order that the orbit of a point be a homeomorphic image of the phase group in a transformation group.

The general reference for definitions is [2]. Throughout this paper $(X, T, \pi)$ will denote a transformation group for which $X$ is a first countable Hausdorff space. The phase group $T$ will be assumed to be generative, that is, $T$ is isomorphic to $C \times R^{m} \times I^{n}$ where $C$ is a compact abelian group, $R$ is the additive group of real numbers, $I$ is the additive group of integers, and $m$ and $n$ are non-negative integers [4]. $P$ will denote a replete semigroup in $T$ which is distinct from $T$. Let $E \subset T$, then $E$ is $P$-extensive provided $E \cap p P \neq \varnothing$ for each $p$ in $P$. Let $x \in X$, then $x$ is $P$-recurrent provided that for each neighborhood $U$ of $x$ there exists a $P$-extensive set $E$ in $T$ such that if $r \in E$ then $x r \in U[1]$. For each $x \in X$ the isotropy subgroup $T_{x}$ of $x$ is the set of all $t \in T$ such that $x t=x$.

Lemma 1. If $x$ is not $P$-recurrent for any replete semigroup $P \neq T$ then the isotropy subgroup $T_{x}$ of $x$ is a subgroup of the compact subgroup of $T$. That is, if $T=C \times R^{m} \times I^{n}$ then $T_{x}=A \times\{0\} \times\{0\}$ where $A \subset C$.

PRoof. For $w \in T$ let $w=\left(w_{1}, w_{2}, w_{3}\right)$ where $w_{1} \in C, w_{2} \in R^{m}$ and $w_{3} \in I^{n}$. We assume that there exists an $a=\left(a_{1}, a_{2}, a_{3}\right) \in T_{x}$ such that not both $a_{2}$ and $a_{3}$ are zero. We consider first the case where $a_{3} \neq 0$. That is, if $a_{3}=\left(a_{3}(1), a_{3}(2), \cdots, a_{3}(n)\right)$ then $a_{3}(j) \neq 0$ for some $j$. Let $P=C \times B$ where $B=\left\{\left(x_{1}, \cdots, x_{m+n}\right) \in R^{m} \times I^{n}: x_{m+j} \geqq 2\right\}$. We first observe that $P$ is a semigroup of $T$ and $P \neq T$.

To see that $P$ is replete let $K$ be a compact subset of $T$. Then there exists a positive number $r$ such that $C \times S(r) \supset K$. $(S(r)$ denotes the sphere of radius $r$ about the origin.) It therefore follows that

$$
\begin{aligned}
& K\left(e^{\prime}, 0, \cdots, r+4,0, \cdots, 0\right) \\
& \quad \subset[C \times S(r)]\left(e^{\prime}, 0, \cdots, r+4,0, \cdots, 0\right) \subset P
\end{aligned}
$$

where $r+4$ appears in the $m+j+1$ position and $e^{\prime}$ denotes the identity in $C$. Thus $P$ is a replete semigroup in $T$.

We now show that $T_{x}$ is $P$-extensive. First, we observe that for $p \in P$ the set $p P=C \times B^{\prime}$ where

$$
B^{\prime}=\left\{\left(x_{1}, \cdots, x_{m+n}\right) \in R^{m} \times I^{n}: x_{m+j} \geqq N\right\}
$$

Received by the editors April 27, 1965. 
for some integer $N$. Also there exists an integer $k$ such that $k a_{3}(j)>N$. Thus if we consider the element $k a=\left(k a_{1}, k a_{2}, k a_{3}\right)$ we have $k a_{1} \in C$, $\left(k a_{2}, k a_{3}\right) \in B^{\prime}$ hence $k a \in p P$. Also since $T_{x}$ is a subgroup of $T$, $k a \in T_{x}$ which implies that $T_{x} \cap p P \neq \varnothing$ for all $p \in P$. Hence $T_{x}$ is $P$ extensive.

For each $t \in T, x t=x$. Therefore, if $U$ is any open set containing $x$ then $x T_{x} \subset U$ which implies $x$ is $P$-recurrent and is a contradiction. The case where $a_{2} \neq 0$ can be handled in the same manner.

THEOREM 1. A necessary and sufficient condition that the mapping $g: T \rightarrow 0(x)$ by $g(t)=x t$ be a homeomorphism is that the isotropy subgroup $T_{x}$ of $T$ at $x$ restricted to the compact group $C$ is trivial and that $x$ is not $P$-recurrent for any replete semigroup $P \neq T$.

Proof. Assume that $g: T \rightarrow 0(x)$ is a homeomorphism. Since $g$ is one-to-one it follows that $T_{x}$ is trivial. In order to show that $x$ is not $P$-recurrent for any replete semigroup of $T$ we assume there exists a replete semigroup $P \neq T$ such that $x$ is $P$-recurrent. Let $\left\{U_{n}\right\}$ be a sequence of open sets such that $x=\cap U_{n}$ and let $A_{n}$ be the corresponding $P$-extensive subsets of $T$.

Let $U$ be an open subset of $C$ which contains $e^{\prime}$, the identity of $C$, and has the property that $U \neq C$. If $S$ is a semigroup of $R^{1}$ which contains some interval containing zero then $S=R^{1}$. Thus, since $P \neq T$, for some $j$ there exists a number $p_{j}$ and an interval $U_{j}$ containing zero such that if $P_{j}$ is the projection of $P$ onto the $j$-axis $(j>1)$ then $p_{j} \in P_{j}$ and $p_{j} P_{j} \cap U_{j}=\varnothing$. Let $K=U \times R^{1} \times \cdots \times U_{j} \times \cdots$ $\times I_{n}$ and $p=\left(x_{1}, \cdots, p_{j}, \cdots, x_{m+n+1}\right) \in P$. Then $p P \cap K=\varnothing$. Thus since $A_{n} \cap p P \neq \varnothing$ for all $n$ we have $A_{n} \cap\{T-K\} \neq \varnothing$ for all integers $n$. Let $r_{n} \in A_{n}-K$ for each $n$. Since $\cap U_{n}=x$ it follows that $\lim _{n \rightarrow \infty} x r_{n}$ $=x e=x$. But since $g$ is a homeomorphism this implies that $\lim _{n \rightarrow \infty} r_{n}$ $=e$ which is clearly impossible. Thus $x$ is not $P$-recurrent for any $P \neq T$.

We now assume that $x$ is not $P$-recurrent for any replete semigroup $P \neq T$. It follows from Lemma 1 that $T_{x} \subset C$. But since $T_{x}$ restricted to $C$ is trivial this means $T_{x}=\left\{\left(e^{\prime}, 0,0\right)\right\}$. This implies that the mapping $g: T \rightarrow 0(x)$ by $g(t)=x t$ is one-to-one. Since $(X, T, \pi)$ is a transformation group it follows that $g$ is continuous. We have only to show that $g^{-1}$ is continuous. In order to do this it is sufficient to show that if $\lim _{n \rightarrow \infty} x t_{n}=x$ then $\lim _{n \rightarrow \infty} t_{n}=e$.

If this is not the case then either there exists a subsequence $\left\{t_{n}^{\prime}\right\}$ of $\left\{t_{n}\right\}$ and an $a=\left(a_{1}, a_{2}, a_{3}\right) \neq\left(e^{\prime}, 0,0\right)$ such that $\lim _{n \rightarrow \infty} t_{n}^{\prime}=a$ or the sequence $\left\{t_{n}\right\}$ intersects the set $T-\{C \times S(r)\}$ for all $r>0$, where $S(r)$ denotes the sphere of radius $r$ about the origin in $R^{m} \times I^{n}$. If 
$\lim _{n \rightarrow \infty} t_{n}^{\prime}=a$ then, since $\pi$ is continuous, it follows that $x a=x$ which is a contradiction since $g$ is one-to-one.

In the second case let $t_{i}=\left(t_{i}(1), \cdots, t_{i}(m+n+1)\right)$ where $\left(t_{i}(2), \cdots, t_{i}(m+n+1)\right) \in R^{m} \times I^{n}$. For some $j>1$ there exists a subsequence of $\left\{t_{i}(j)\right\}$ which converges either to positive or negative infinity. We consider the case where some subsequence of $\left\{t_{i}(j)\right\}$ converges to positive infinity and denote by $t_{i}^{\prime}$ the corresponding elements of $\left\{t_{i}\right\}$. The case where some subsequence of $\left\{t_{i}(j)\right\}$ converges to negative infinity can be handled in the same manner. If we let $P=C \times B$ where $B=\left\{\left(x_{1}, \cdots, x_{m+n}\right) \in R^{m} \times I^{n}: x_{j} \geqq 2\right\}$ then $P$ is a replete semigroup of $T$ and $\left\{t_{i}^{\prime}\right\}$ is a $P$-extensive subset of $T$. It follows that $x$ is $P$-recurrent, which is impossible.

We observe that if $m=n=0$ then $T=C$. Since there are no replete semigroups of $T$ other than $T$ itself the notion of $P$-recurrence is not meaningful. In this case Theorem 1 reduces to the remarks found in $[3$, p. 65].

\section{BIBLIOGRAPHY}

1. J. D. Baum, P-recurrence in topological dynamics, Proc. Amer. Math. Soc. 7 (1956), 1146-1154.

2. W. H. Gottschalk, and G. A. Hedlund, Topological dynamics, Amer. Math. Soc. Colloq. Publ. Vol. 36. Amer. Math. Soc., Providence, R. I., 1955.

3. D. Montgomery and L. Zippin, Topological transformation groups, Interscience, New York, 1955.

4. A. Weil, L'intégration dans les groupes topologiques et ses applications, Actualités Sci. Ind., No. 869, Paris, 1940.

UNIVERSITY OF VIRGINIA 